\title{
Using a social platform as an internal communication tool A case study
}

n 2017, the Karolinska Institutet University Library conducted a project to replace the existing Intranet with a new improved tool. At the start of the project, we assumed it would result in a new, improved version of the existing Intranet. However, the project group eventually decided to replace the original Intranet with a combination of three tools that we believed would better meet the library staff's needs.

This article will focus on the most controversial of the three tools: Workplace, which is a communication tool produced by Facebook. We will discuss the reasoning and deliberation prior to adopting the tool, the implementation process, and finally an evaluation of whether it has met the goals and expectations of the library a year after implementation. In summary, our experience with Workplace has been mixed, but the positive aspects have outweighed the negative.

\section{Background}

Karolinska Institutet is Sweden's largest medical university and research organization and is composed of two campus areas, each of which has a library. The library staff is composed of slightly more than 100 people, who support the university with traditional library services as well as additional services like bibliometrics, student IT support, and academic writing support.
A project group was formed in 2017 to replace the existing library Intranet. As a starting point, the group was given documentation from a previous project group that had made an "impact map" of the library's internal communication needs. Impact mapping is a technique used to elicit goals from users and their needs. ${ }^{1}$ In our case, the mapping resulted in five goals related to internal communication. For example, one goal was to increase transparency between departments and co-workers.

The group came to the conclusion that it would be difficult to successfully meet all the goals with a single Intranet tool, so we decided to pursue a solution with three separate tools:

- one for static information like policies, guidelines, rules, etc.;

- one FAQ website, which would be used by co-workers providing user support; and

- one communication tool for communication with the entire library or within a working group.

For the communication tool, the group eventually decided to test Workplace. Work-

Glenn Haya is librarian, email: glenn.haya@ki.se, Sara M. Lind is librarian, email: sara.m.lind@ki.se, and Sara Janzen is librarian, email: sara.janzen@ki.se, at Karolinska Institutet University Library

(c) 2020 Glenn Haya, Sara M. Lind, and Sara Janzen 
place is a web-based collaboration and communication tool produced by Facebook. The premium version is sold as a service to private companies but is freely available for educational institutions. The tool looks, feels, and functions much like traditional Facebook. However, it is a separate product, and there is no connection between an employee's private Facebook account and Workplace account. The most important distinction between Workplace and Facebook is that Workplace is free from advertisements, and the organization owns its own data.

Workplace seemed to offer clear benefits, the primary one being that since the interface is almost identical to Facebook's, it was already intuitive and familiar to the majority of our staff. Also, the interface and mobile app were far better than we would have been able to achieve if we had tried to build a custom site in-house including tools such as live video streaming and video chat. Finally, it was free for educational institutions.

However, we also had a number of reservations about Workplace from the start. How likely was it that Facebook would abandon Workplace a year or two down the line? Also, we worried that co-workers would miss important information in a chaotic Facebook-like feed. Finally, we wondered if our Co-workers and management would reject the solution simply because it came from Facebook.

\section{Testing}

Before coming with a final recommendation, we did a several months-long pilot test with two of our library departments, asking them to use Workplace as their primary communication tool, and then gathering feedback from them on what worked well and what worked poorly. We held focus groups with the departments and with our management team to get feedback from them.

We also tried to engage staff by holding open demos every few weeks, where any interested staff member could come and listen to what the group was working on and ask questions.
In order to prepare the feedback we received for library management, we divided the responses into "hopes" and "fears" about Workplace.

Our co-workers' hopes centered around the tool's potential to increase transparency across library departments and increase engagement and participation across the whole library. Since the interface was familiar to many, people anticipated that there would be a low barrier to entry, resulting in the entire staff participating and actively communicating with each other.

On the other hand, the biggest fears our staff had with Workplace is that there would be too much information to deal with, and it would be chaotic. For example, individual posts could easily be lost in the feed, and there was no way of guaranteeing that an important post would be read by all the relevant co-workers. Some staff feared these problems would lead to confusion and stress.

The positives and negatives were presented to the library's management, who then decided to go forward with the implementation of Workplace, since the potential positive outcomes were deemed to outweigh the potential negatives ones. In addition, the decision was made with the belief that we could implement best practices that would mitigate some of the fears expressed by coworkers during the implementation phase.

\section{Implementation}

The implementation of Workplace was helped by the fact that the project group had been evaluating the tool for a long period of time and had made efforts to be transparent during the process.

Having already identified the misgivings of the staff regarding Workplace, the project group tried to mitigate them by providing a policy and guidelines document where, among other things, there was specific information about:

- what type of content can be published on Workplace,

- which groups were mandatory for all staff to be a member of and monitor (staff 
members were also added to these groups by default),

- recommendations for settings regarding notifications, and

- recommendation for creating new groups.

In addition to the policy and guidelines, a Workplace group was created for users to post

Workplacerelated questions. Finally, the project group offered drop in times for people to come and ask questions in person, if needed.

\section{Usage}

Usage of Workplace versus the previous Intranet.

Dra matic

increase in

\section{content and interaction}

The graph above shows a week's activity in the previous Intranet compared to Workplace (we took as a sample the second week of May 2017 versus the same week in 2018). The amount of content published in Workplace is far greater than the content published in our previous Intranet. Even more dramatic is the increase in interactions in the form of comments. Though the ability to comment was available in the previous Intranet, it was rarely used, whereas it is used often on Workplace.

The dramatic difference is partially explained by the fact that the previous Intranet was used for communicating with the entire library staff, whereas small working or project groups communicated with each other using Slack. After we implemented Workplace, much of that small group communication has moved over to Workplace. However, this alone cannot account for the entire increase. Using
Workplace has clearly resulted in people sharing more information and interacting more with each other online.

\section{Examples of co-workers using Workplace in new ways}

After a few weeks of usage, we began to see staff use Workplace in ways that were not possible using our previous Intranet.

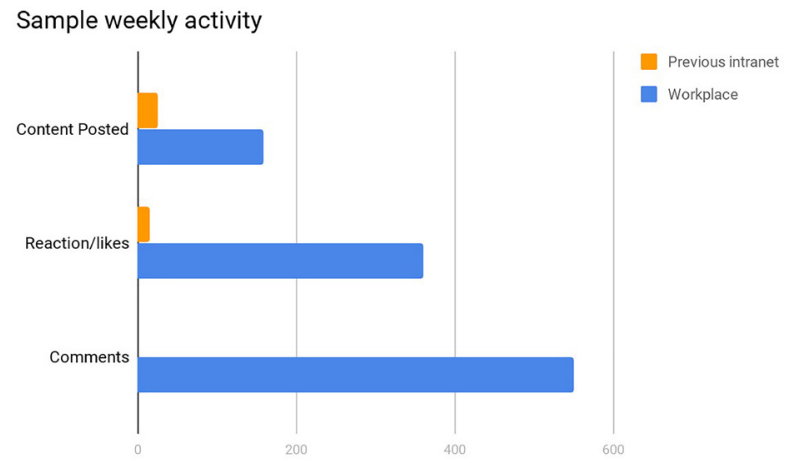

Live video streaming The possibility of live video streaming has allowed co-workers to broadcast events, such as workshops, so those who cannot physically be present can still participate and even interact.

A typical example of this is a biweekly meeting where the library management group sends a representative to give library staff the latest news. This is now broadcast live to the whole library staff, so those who are interested but cannot attend can view the meeting and pose questions as comments.

Streaming has been a popular service. A staff survey discussed in detail that $85 \%$ of respondents said they have benefitted from the streaming functionality in Workplace.

\section{Embedded videos and webpages}

While embedding was possible in the previous Intranet, it is easier and better implemented on Workplace and, as a result, is used much more often. Below is an example of a colleague calling attention to a page on our library website.

Secret groups with RSS feeds

Workplace supports "secret" groups, 
which simply means groups not visible to the entire staff. Some co-workers have used this function to create their own individual group that only they have access to, which they use, for example, as an RSS reader or to write individual notes for themselves.

\section{Measuring staff satisfaction}

Approximately one year after we implemented Workplace, we conducted a staff survey to help us evaluate the effects of all three tools that function as the library's new Intranet. Response rates were high. We received 72 responses (approximately $70 \%$ of our staff) and a total of 392 written comments.

In general, the staff was satisfied with Workplace, with $68 \%$ of respondents saying they thought the overall effects of implementing Workplace have been positive. In addition, 84\% said they thought that using Workplace has resulted in increased transparency between employees working in different departments.

The issue of too much information remains a significant concern, with 32\% responding that they thought too much was published there. We received comments such as ". . . there can often be too much noise on Workplace which makes it easy to miss individual posts" and "I feel a responsibility to read/skim what is published on Workplace and it takes time."

Our survey indicates that the majority of our co-workers have a positive view of Workplace. However, Facebook as a company has been the center of numerous questions and controversies, ${ }^{2}$ and some of the staff's negative feelings about Facebook transfer over to Workplace. This factor is at least worth considering when choosing a communications tool.

\section{Conclusion}

In conclusion, Workplace has been a net benefit to the library and has helped us achieve our goal of more transparency between colleagues working in different departments, campuses, and projects. The choice to implement Workplace still seems reasonable more than one year after the decision was made, and the library has no current plans to explore other alternatives.

Libraries interested in increasing transparency in their organization should consider Workplace, though they may also want to also consider any open source alternatives to the social media platform, if they want to avoid any controversy regarding the company behind the tool.

\section{Notes}

1. Ingrid Ottersten, Effect Managing IT (Copenhagen Business School Press, 2007)

2. "Critcism of Facebook," Wikipedia, accessed May 13, 2019, https://en.wikipedia. org/wiki/Criticism_of_Facebook. „2 